\title{
DENSE MORPHISMS IN COMMUTATIVE BANACH ALGEBRAS
}

\author{
GUSTAVO CORACH AND FERNANDO DANIEL SUÁREZ
}

\begin{abstract}
Using a new notion of stability we compute exactly the stable rank of the polydisc algebra, extend Oka's extension theorem to $n$-tuples of functions without common zeros and give an estimation for a question raised by Swan concerning the stable rank of a dense subalgebra of a given Banach algebra.
\end{abstract}

0. Introduction. The stable rank of a ring $A$ [2] is an algebraic invariant of $A$ which turns out to be closely related to the topology of the spectrum of $A$ when $A$ is a complex commutative Banach algebra (see $[4,7,8])$. In this paper we study certain approximation and interpolation problems which are related to that notion. Our main result (Theorem 2.7), whose statement is too technical to be described here, has several applications. First, we find exactly the stable rank of the polydisc algebra $A_{n}$ of $C^{n}: \operatorname{sr}\left(A_{n}\right)=[n / 2]+1$. Moreover, for every $n$-generated algebra $A, \operatorname{sr}(A) \leqslant$ $[n / 2]+1$ and the equality holds if the joint spectrum of a system of $n$ generators of $A$ has nonvoid interior in $C^{n}$. Next, we obtain a result in connection with an old problem of Cartan [3] who looked for conditions on an $r$-tuple of holomorphic functions on a domain $\Omega$ in $\mathbf{C}^{k}$ without common zero to be completed to an invertible matrix of such functions. This problem has been considered by Lin [16] and Sibony and Wermer [20]. We prove that if $A$ is $n$-generated then every $m$-tuple $a=\left(a_{1}, \ldots, a_{m}\right)$, such that $\hat{a}_{1}, \ldots, \hat{a}_{m}$ have no common zero (see Definitions below), can be completed to an invertible matrix with entries in $A$ for $m \geqslant[n / 2]+1$. Another application is a $k$-dimensional version of Oka's extension theorem.

Finally, we give a partial answer to a question of Swan [23, Remark, p. 206] if there exists a morphism $f: A \rightarrow B$ with dense image such that " $f(a)$ invertible $\Rightarrow a$ invertible" then $\operatorname{sr}(A) \leqslant \operatorname{sr}(B) \leqslant \operatorname{sr}(A)+2$.

Preliminaries. In this paper Banach algebra means a complex commutative Banach algebra with identity. The spectrum of a Banach algebra $A$ is the space $X(A)$ of all nonzero complex homomorphisms of $A$; the elements of $X(A)$, called characters, are continuous and $X(A)$ is a compact Hausdorff space with the induced weak* topology. The Gelfand transformation ${ }^{\wedge}: A \rightarrow C(X(A))$ is defined by $\hat{a}(h)=h(a)(a \in A, h \in X(A))$. For any $n$-tuple $a=\left(a_{1}, \ldots, a_{n}\right) \in A^{n}$ we write $\hat{a}=\left(\hat{a}_{1}, \ldots, \hat{a}_{n}\right): X(A) \rightarrow \mathbf{C}^{n}$; the image of $\hat{a}$ is the joint spectrum of $a$, which we denote $\sigma(a)$ and we recall that $\sigma(a)=\left\{\lambda=\left(\lambda_{1}, \ldots, \lambda_{n}\right) \in \mathbf{C}^{n}: \sum_{i=1}^{n} A \cdot\left(a_{i}-\lambda_{i}\right)\right.$ $\neq A\}$.

Received by the editors November 18, 1986.

1980 Mathematics Subject Classification (1985 Revision). Primary 46J15, 18F25; Secondary 32E20, 32E25, 55Q55. 
If $a=\left(a_{i}\right)_{i \in I}$ is a (possibly infinite) system of generators of $A$ and $\sigma(a)=$ $\left\{\left(h\left(a_{i}\right)\right)_{i \in I} \in \mathbf{C}^{I}: h \in X(A)\right\}$, then $\sigma(a)$ is a polynomially convex compact subset of $\mathbf{C}^{I}$ (where a compact subset $X$ of $\mathbf{C}^{I}$ is polynomially convex if it coincides with its hull $\hat{X}=\left\{z \in \mathbf{C}^{l}:|p(z)| \leqslant \sup \{|p(x)|: x \in X\}\right.$ for every polynomial $\left.p\right\}$ [22]). Moreover $\hat{a}: X(A) \rightarrow \sigma(a)$ is a homeomorphism. For a compact subset $X$ of $\mathbf{C}^{I}$ we define $P(X)$ as the closure, in $C(X)$, of the polynomial functions. The spectrum of $P(X)$ is identified to $\hat{X}$. Observe that there exists a continuous homomorphism $A \rightarrow P(\sigma(a))$ (where $a=\left(a_{i}\right)$ is a system of generators), with dense image. Sometimes we identify $X(A)$ to $\sigma(a)$ and write $P(X(A))$ instead of $P(\sigma(a))$.

We put $U_{n}(A)=\left\{a \in A^{n}: 0 \notin \sigma(a)\right\}$; its elements are called unimodulars. A unimodular $\left\{a_{1}, \ldots, a_{n-1}, a_{n}\right)$ is reducible if there exist $x_{1}, \ldots, x_{n-1}$ in $A$ such that $\left(a_{1}+x_{1} a_{n}, \ldots, a_{n-1}+x_{n-1} a_{n}\right)$ is unimodular.

The stable rank of $A$ is the least $n$ such that every $a \in U_{n+1}(A)$ is reducible.

We use the symbol $\Delta$ for the closed unit disc of the complex plane $C$. Given spaces $X, Y, C(X, Y)$ denotes the set of all maps from $X$ into $Y$.

Definition 1.1. Let $E, B$, and $X$ be Hausdorff topological spaces. Suppose that $B$ is metrizable with a metric $d$ and $X$ is a compact space. A map $p: E \rightarrow B$ has property $(\mathrm{H})$ with respect to $X$ if for every commutative diagram

$$
\begin{array}{ccc}
X & \rightarrow & E \\
i \downarrow & & \downarrow p \\
I \times X & \rightarrow \vec{f} & B
\end{array} \quad(I=[0,1], i(x)=(0, x))
$$

and $\varepsilon>0$ there exists a map $F: I \times X \rightarrow E$ such that $F i=f$ and $\sup \{d(p F(t, x), \tilde{f}(t, x)): t \in I, x \in X\} \leqslant \varepsilon$. A map $p: E \rightarrow B$ is a Serre quasifibration if it has property $(\mathrm{H})$ with respect to every cube $I^{m}(m \geqslant 0)$. When $\varepsilon=0$ we get the classical notion of Serre fibration (see [14, pp. 61-64]).

Proposition 1.2. Let $\phi: A \rightarrow B$ be a dense morphism of Banach algebras (i.e. $\phi$ is a continuous homomorphism with dense image). Consider the induced group homomorphism $\phi: \mathrm{GL}_{n}(A) \rightarrow \mathrm{GL}_{n}(B)$, with image $L$.

(1) for $b \in \mathrm{GL}_{n}(B), b \in \bar{L}$. (the closure of $L$ in $\mathrm{GL}_{n}(B)$ ) if and only if there exists $b^{\prime} \in L$ which belongs to the connected component of $b$ in $\mathrm{GL}_{n}(B)$;

(2) $\phi: \mathrm{GL}_{n}(A) \rightarrow \mathrm{GL}_{n}(B)$ is a quasi-fibration.

Proof. $(1)(\Rightarrow)$ Let $b \in \bar{L}$; then there exists $a \in \mathrm{GL}_{n}(A)$ such that $\|\phi(a)-b\|<$ $\left\|b^{-1}\right\|^{-1}$ and $b+t(\phi(a)-b)(t \in I)$ defines an arc in $\mathrm{GL}_{n}(B)$ joining $b$ and $\phi(a)$ : in fact,

$$
b^{-1}(b+t(\phi(a)-b))=1+t b^{-1}(\phi(a)-b)
$$

and

$$
\left\|b^{-1}(\phi(a)-b)\right\|<1 \quad(t \in I) .
$$

$(\Leftarrow)$ We prove first that $\phi\left(\mathrm{GL}_{n}(A)_{0}\right)$ is dense in $\mathrm{GL}_{n}(B)_{0}$ (where in general $G_{0}$ is the connected component of the neutral element); if $y \in \mathrm{GL}_{n}(B)_{0}$ then there exist $b_{1}, \ldots, b_{s} \in M_{n}(B)$ with $y=\exp \left(b_{1}\right) \cdots \exp \left(b_{s}\right)[18$, Chapter I]. Using the continuity of exp and the density of the image of $\phi$, we can approach $y$ by 
$\exp \phi\left(a_{1}\right) \cdots \exp \phi\left(a_{s}\right)=\phi\left(\exp \left(a_{1}\right) \cdots \exp \left(a_{s}\right)\right) \in \phi\left(\mathrm{GL}_{n}(A)_{0}\right)$ for some $a_{1}, \ldots, a_{s} \in M_{n}(A)$; this proves our assertion.

Now, if $G_{1}$ is a connected component of $\mathrm{GL}_{n}(B)$ and $G_{0}=\mathrm{GL}_{n}(B)_{0}$, for $u \in G_{1}$ the translation $x \rightarrow x u$ defines a homomorphism $G_{0} \rightarrow G_{1}$ whose inverse map is $y \rightarrow y u^{-1}$. Then, if $b \in G_{1}$ and $b^{\prime} \in G_{1} \cap L$ we get $b\left(b^{\prime}\right)^{-1} \in G_{0} \subset \bar{L}$, but $b^{\prime} \in \bar{L}$ which is a subgroup of $\mathrm{GL}_{n}(B)$, thus $b=b\left(b^{\prime}\right)^{-1} b^{\prime} \in \bar{L}$, too.

(2) Firstly, we prove that $\phi: \mathrm{GL}_{n}(A) \rightarrow \mathrm{GL}_{n}(B)$ has property $(\mathrm{H})$ with respect to $I^{0}=\{0\}$.

For this, we consider the commutative diagram

$$
\begin{array}{cccc}
\{0\} & \stackrel{f}{ } & \mathrm{GL}_{n}(A) & \\
i \downarrow & & \downarrow \phi & (i(0)=(0,0)) \\
I \times\{0\} & \underset{f}{\rightarrow} & \mathrm{GL}_{n}(B) &
\end{array}
$$

and we look for a map $F: I \times\{0\} \rightarrow \mathrm{GL}_{n}(A)$ such that $F(0,0)=f(0)$ and $\sup \{\|\phi(F(t, 0))-\tilde{f}(t, 0)\|: t \in I\}<\varepsilon$ for a given $\varepsilon>0$ (where the norm is that of $M_{n}(B)$, which is induced by that of $\left.B\right)$. Observe that we can think of $\tilde{f}$ and $\tilde{f}(0,0)$ as elements of $\mathrm{GL}_{n}(C(I, B))$; moreover, they belong to the some connected component of $\mathrm{GL}_{n}(C(I, B))$, for $s \rightarrow \tilde{f}(s t, 0)$ is an arc which begins at $\tilde{f}(0,0)$ and ends at $\tilde{f}$. Observe also that $\phi: A \rightarrow B$ induces a dense morphism $\varphi: C(I, A) \rightarrow C(I, B)$ which induces, as $\phi$ before, a group homomorphism $\mathrm{GL}_{n}(C(I, A)) \rightarrow \mathrm{GL}_{n}(C(I, B))$, which we denote again $\varphi$. It is clear that $\tilde{f}(0,0)=\phi(f(0))$ belongs to $\varphi\left(\mathrm{GL}_{n}(C(I, A))\right)$. By the remarks above and part (1), there exists $a \in \mathrm{GL}_{n}(C(I, A))$ such that $\|\varphi(a)-\tilde{f}\|<\varepsilon^{\prime}$ for a given $\varepsilon^{\prime}>0$. We shall prove that, for $\varepsilon^{\prime}$ small enough, $F=f(0) a(0)^{-1} a$ is the map we look for (where we are identifying $I$ with $I \times\{0\}$ and looking at $\left.\tilde{f}, a: I \times\{0\} \rightarrow \mathrm{GL}_{n}(A)\right)$. For this, keep $t \in I$ fixed; then

$$
\begin{aligned}
\| \phi( & \left.f(0) a(0)^{-1} a(t)\right)-\tilde{f}(t) \| \\
& \leqslant\left\|\phi\left(f(0) a(0)^{-1} a(t)\right)-\phi(a(t))\right\|+\|\phi(a(t))-\tilde{f}(t)\| \\
& \leqslant\left\|\phi\left(f(0) a(0)^{-1}\right)-1\right\|\|\phi(a(t))\|+\|\varphi(a)-\tilde{f}\|_{M_{n}} \\
& <\left\|\phi\left(f(0) a(0)^{-1}\right)-1\right\|(\|\phi(a(t))-\tilde{f}(t)\|+\|\tilde{f}(t)\|)+\varepsilon^{\prime} \\
& <\left\|\phi\left(f(0) a(0)^{-1}\right)-1\right\|\left(\varepsilon^{\prime}+\|\tilde{f}(t)\|\right)+\varepsilon^{\prime}
\end{aligned}
$$

where

$$
\left\|\phi\left(f(0) a(0)^{-1}\right)-1\right\| \leqslant\|\phi(f(0))\| \cdot\left\|\phi\left(a(0)^{-1}\right)-\phi\left(f(0)^{-1}\right)\right\| .
$$

If we put $\beta=\left\|\phi\left(a(0)^{-1}\right)-\phi\left(f(0)^{-1}\right)\right\|$, we get

$$
\begin{aligned}
\beta & \leqslant\left\|\phi\left(a(0)^{-1}\right)\right\|\left\|\phi\left(f(0)^{-1}\right)\right\|\|\phi(a(0))-\phi(f(0))\| \\
& \leqslant\left(\beta+\left\|\phi\left(f(0)^{-1}\right)\right\|\right)\left\|\varphi\left(f(0)^{-1}\right)\right\|{\varepsilon^{\prime}}^{\prime}
\end{aligned}
$$


Thus $\beta \leqslant 2\left\|\phi\left(f(0)^{-1}\right)\right\|^{2} \varepsilon^{\prime}$ choosing $0<\varepsilon^{\prime}<\frac{1}{2}\left\|\phi\left(f(0)^{-1}\right)\right\|$. Replacing this estimation in the inequalities above we get

$$
\begin{aligned}
\left\|\phi\left(f(0) a(0)^{-1} a(t)\right)-\tilde{f}(t)\right\| & \leqslant\|\phi(f(0))\| 2\left\|\phi\left(f(0)^{-1}\right)\right\|^{2} \varepsilon^{\prime}\left(\|\tilde{f}(t)\|+\varepsilon^{\prime}\right)+\varepsilon^{\prime} \\
& =\left[2\|\phi(f(0))\|\left\|\phi\left(f(0)^{-1}\right)\right\|^{2}\left(\|\tilde{f}(t)\|+\varepsilon^{\prime}\right)+1\right] \varepsilon^{\prime} .
\end{aligned}
$$

Now, $\|\tilde{f}(t)\| \leqslant\|\tilde{f}\|_{M_{n}(C(I, B))}$ so we can choose $\varepsilon^{\prime}>0$ such that the whole expression is less than $\varepsilon$. Then, for every $t \in I,\left\|\phi\left(f(0) a(0)^{-1} a(t)\right)-\tilde{f}(t)\right\|<\varepsilon$, as claimed.

Next, we must prove that the map $\phi: \mathrm{GL}_{n}(A) \rightarrow \mathrm{GL}_{n}(B)$ has property $(\mathrm{H})$ with respect to $I^{m}$ for every positive integer $m$; but we can reduce the general case to the above situation by observing that the commutative diagram

$$
\begin{aligned}
& I^{m} \stackrel{f}{\rightarrow} \mathrm{GL}_{n}(A) \\
& i \downarrow \quad \downarrow \phi \quad i(q)=(0, q)
\end{aligned}
$$

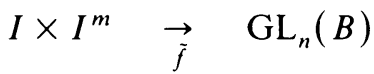

can be transformed into

$$
\begin{array}{cccc}
\{0\} & \stackrel{g}{\rightarrow} & \mathrm{GL}_{n}\left(C\left(I^{m}, A\right)\right) \\
i \downarrow & & \downarrow \varphi \\
I \times\{0\} & \rightarrow & \mathrm{GL}_{n}\left(C\left(I^{m}, B\right)\right)
\end{array}
$$

where $g(0)=f$ and $\tilde{g}(t, 0)=\tilde{f}_{t}$.

Corollary 1.3. If $\phi: A \rightarrow B$ is a dense morphism of Banach algebras then the induced map $\phi^{\prime}: U_{n}(A) \rightarrow U_{n}(B)$ is a quasifibration.

Proof. As before, it suffices to prove that $\phi^{\prime}$ has property $(\mathrm{H})$ with respect to $I^{0}=\{0\}$.

Let $a \in U_{n}(A), b=\phi^{\prime}(a) \in U_{n}(B), \gamma: I \rightarrow U_{n}(B)$ be an arc beginning at $b$, and $\varepsilon>0$.

Consider the commutative diagram:

$$
\begin{array}{cccrc}
\tau & \mathrm{GL}_{n}(A) & \stackrel{\phi}{\rightarrow} & \mathrm{GL}_{n}(B) & \sigma \\
\downarrow & t_{a} \downarrow & & \downarrow t_{b} & \downarrow \\
\tau \cdot a & U_{n}(A) & \rightarrow & U_{n}(B) & \sigma \cdot b
\end{array}
$$

It is known that $t_{b}$ is a Serre fibration $[4,6]$ so there exists an arc $\gamma_{1}: I \rightarrow \mathrm{GL}_{n}(B)$ such that $\gamma_{1}(0)=1$ and $t_{b} \circ \gamma_{1}=\gamma$. Now, using the fact that $\phi$ is a quasi-fibration, we find an arc $\gamma_{2}: I \rightarrow \mathrm{GL}_{n}(A)$ such that $\gamma_{2}(0)=1$ and $\left\|\phi\left(\gamma_{2}(t)\right)-\gamma_{1}(t)\right\|<\varepsilon /\|b\|$ $(t \in I)$. It is easy to see that $\tilde{\gamma}=t_{a} \circ \gamma_{2}$ verifies $\tilde{\gamma}(0)=a$ and $\left\|\phi^{\prime}(\tilde{\gamma}(t))-\gamma(t)\right\|<\varepsilon$ for every $t \in I$.

REMARK 1.4. For $\phi$ a surjective morphism, an analogous (and easier) proof shows that $\phi: \mathrm{GL}_{n}(A) \rightarrow \mathrm{GL}_{n}(B)$ and $\phi^{\prime}: U_{n}(A) \rightarrow U_{n}(B)$ are not only quasi-fibrations but also Serre fibrations. The first result is well-known theorem of Michael [17]. If $A$ 
is a complex commutative Banach algebra with spectrum $X(A)$ then the ArensNovodvorski-Taylor theory [24] identifies the set of connected components of $U_{n}(A)$ with the set of homotopy classes of maps $X(A) \rightarrow C_{*}^{n}$ : in symbols, $\pi_{0}\left(U_{n}(A)\right)=$ $\left[X(A), C_{*}^{n}\right]\left[X(A), S^{2 n-1}\right]$ (where $S^{k}$ is the unit sphere of $R^{k+1}$ ). Observe that the transpose of a morphism $f: A \rightarrow B, f^{*}: X(B) \rightarrow X(A)$, induces a map $\left[X(A), S^{k}\right]$ $\rightarrow\left[X(B), S^{k}\right]$. By combining the above results we get the following:

Corollary 1.5. Let $f: A \rightarrow B$ be a morphism of Banach algebras.

(1) If $f$ is dense, then the induced map $f: U_{k}(A) \rightarrow U_{k}(B)$ has dense image if and only if the mapping $\left[X(A), S^{2 k-1}\right] \rightarrow\left[X(B), S^{2 k-1}\right]$ is surjective.

(2) If $f$ is surjective, then $f: U_{k}(A) \rightarrow U_{k}(B)$ is surjective if and only if $\left[X(A), S^{2 k-1}\right] \rightarrow\left[X(B), S^{2 k-1}\right]$ is surjective.

2. We recall two definitions. A closed subset $F$ of $X(A)$ is a hull if there exists $E \subset A$ such that hull $(E)=\{h \in X(A): h(a)=0(a \in E)\}=F$. For a closed subset $F$ of $X(A)$ we put $\hat{F}=\left\{h \in X(A):|h(a)| \leqslant\|\hat{a}\|_{F}(a \in A)\right\}$. $F$ is $A$-convex if $\hat{F}=F$. It is clear that every hull is $A$-convex, but the converse fails, in general.

Proposition 2.1. Let $f: A \rightarrow B$ be a dense morphism. Then the transpose $f^{*}$ : $X(B) \rightarrow X(A)$ is injective and its image $f^{*}(X(B))$ is $A$-convex; moreover, if $f$ is onto then $f^{*}(X(B))$ is a hull.

For the proof, we need the following result, which follows easily [22, Theorem 5.8].

LEMmA 2.2. Let $A$ be a complex commutative Banach algebra and $\left\{a_{i}: i \in I\right\}$ a system of generators of $A$. Then $\hat{a}=\left(\hat{a}_{i}: i \in I\right): X(A) \rightarrow \sigma(a) \subset C^{I}$ is a homeomorphism, $\sigma(a)$ is polynomially convex, and if $R \subset \sigma(a)$ is polynomially convex then $\hat{a}^{-1}(R) \subset X(A)$ is A-convex. Conversely, if $F$ is $A$-convex then $\hat{a}(F)$ is polynomially convex.

Proof of the Proposition. Let $a=\left(a_{i}: i \in I\right)$ be a system of generators of $A$. Then $f(a)=\left(f\left(a_{i}\right): i \in I\right)$ generates $B$ and $\sigma(f(a)) \subset \sigma(a)$ is a polynomially convex compact subset of $C^{I}$ and $f^{*}(X(B))=\hat{a}^{-1}(\sigma(f(a))) \subset X(A)$ is $A$-convex, by the lemma. If $f$ is onto then $B$ can be identified with $A / \operatorname{ker} f$, so $X(B) \approx$ $X(A / \operatorname{ker} f) \approx \operatorname{hull}(\operatorname{ker} f)$ and the second assertion follows easily.

We need the following result of Duchamp and Stout [11, p. 53] which is a consequence of a classical result of Andreotti and Narasimhan [1].

LEMMA 2.3. Let $T$ be a polynomially convex compact subset of $C^{n}$. Then for $k \geqslant n$ the Čech cohomology groups $H^{k}(T)$ with integer coefficients are trivial.

THEOREM 2.4. Let $Y \subset X$ be two polynomially convex compact subsets of $C^{n}$. Then for $k \geqslant n$ the restriction induces a surjective mapping $\left[X, S^{k}\right] \rightarrow\left[Y, S^{k}\right]$.

Proof. We consider, first, the case when $X=P$ and $Y=Q$ are polynomial polyhedra [12, p. 66]. Then $P$ and $Q$ are triangulable and $H^{m}(P)=H^{m}(Q)=0$ $(m \geqslant 2 n-1)$ so $[14$, p. 228] if $i$ denotes the inclusion $Q \subset P$ then the following conditions are equivalent:

(1) $i^{*}: H^{k}(P) \rightarrow H^{k}(Q)$ is an isomorphism for $k>n$ and an epimorphism for $k=n$; 
(2) $i^{*}: \pi^{k}(P) \rightarrow \pi^{k}(Q)$ is an isomorphism for $k>n$ and an epimorphism for $k=n$ (where $\pi^{k}(T)$ is the $k$ th cohomotopy group of $T$ ). By the lemma above, $H^{k}(P)=H^{k}(Q)=0$ for $k \geqslant n$, so (2) holds and we get that $\left[P, S^{k}\right] \rightarrow\left[Q, S^{k}\right]$ is onto for $k \geqslant n$.

We consider now the general case. We must prove that for every map $f$ : $Y \rightarrow \mathbf{R}_{*}^{k+1}\left(\mathbf{R}_{*}^{k+1}=\mathbf{R}^{k+1} \backslash\{0\}\right)$ there exists an extension of $f, \quad F: X \rightarrow \mathbf{R}_{*}^{k+1}$ $(k \geqslant n)$. Let $P$ be a $p$-polyhedron such that $P \supset X$ and extend $f: P \rightarrow \mathbf{R}^{k+1}$; let $Q \subset P$ be a $p$-polyhedron such that $Q \supset Y$ and $f(Q) \subset \mathbf{R}_{*}^{k+1}$; by the first case, $f \mid Q$ admits an extension $G: P \rightarrow \mathbf{R}_{*}^{k+1}$ and then $F=G \mid X$ verifies $F(X) \subset \mathbf{R}_{*}^{k+1}$ and $F|Y=f| Y$.

In the following we use the notation $s_{n}=[n / 2]+1$.

COROllary 2.5. Let $Y \subset X$ be two polynomially convex compact subsets of $\mathbf{C}^{n}$. Then the mapping $\left[X, \mathbf{C}_{*}^{k}\right] \rightarrow\left[Y, \mathbf{C}_{*}^{k}\right]$ is onto for every $k \geqslant s_{n}$.

Proof. It suffices to observe that $2 k-1 \geqslant n$ for $k \geqslant s_{n}$ and use the theorem.

COROLlary 2.6. Let $Y$ be a polynomially convex compact subset of $\mathbf{C}^{n}$. Then $\left[Y, \mathbf{C}_{*}^{k}\right]$ is trivial for $k \geqslant s_{n}$.

Proof. Take a ball $X$ containing $Y$ and use the corollary above.

MaIn Theorem 2.7. Let $f: A \rightarrow B$ be a homomorphism of Banach algebras and suppose that $B$ is $n$-generated.

(1) If $f$ is onto, the induced map $U_{k}(A) \rightarrow U_{k}(B)$ is onto for $k \geqslant s_{n}$.

(2) If $f$ is a dense morphism, then the induced map $U_{k}(A) \rightarrow U_{k}(B)$ has dense image for $k \geqslant s_{n}$.

Proof. Let $b_{1}, \ldots, b_{n} \in B$ be generators of $B$. It is well known $[\mathbf{1 2}, \mathbf{2 2}]$ that $X(B)$ is homeomorphic to the joint spectrum $\sigma_{B}\left(b_{1}, \ldots, b_{n}\right)$, which is a polynomially convex compact subset of $C^{n}$. Then, for $k \geqslant s_{n}$,

$$
\pi_{0}\left(U_{k}(B)\right)=\left[X(B), C_{*}^{k}\right]=\left[\sigma_{B}\left(b_{1}, \ldots, b_{n}\right), C_{*}^{k}\right]
$$

is trivial for the last corollary and the result follows from Corollary 1.5 .

REMARK 2.8. The proof shows that $U_{k}(B)$ is connected for $k \geqslant s_{n}$.

3. Applications. The rest of the paper is devoted to applications of the Main Theorem.

The dense stable rank. It is well known $[4,8]$ that if $A$ is a commutative ring with identity then $\operatorname{sr}(A) \leqslant n$ iff for every closed ideal $I$ of $A$ the induced mapping $U_{n}(A) \rightarrow U_{n}(A / I)$ is onto. In other words, $\operatorname{sr}(A) \leqslant n$ iff for every surjective morphism $f: A \rightarrow B, U_{n}(A) \rightarrow U_{n}(B)$ is onto. This statement yields to the following notion of stability.

Definition 3.1. The dense stable rank of a Banach algebra $A$, denoted by $\operatorname{dsr}(A)$, is the least integer $n$ such that for every dense morphism $f: A \rightarrow B$, the induced map $U_{n}(A) \rightarrow U_{n}(B)$ has dense image. We write $\operatorname{dsr}(A)=+\infty$ if there exists no such $n$. It is obvious that $\operatorname{sr}(A) \leqslant \operatorname{dsr}(A)$, but we do not know if equality holds for every commutative Banach algebra $A$. This question is related to a problem raised by Swan [23]. We will discuss this later. 
A Banach algebra $A$ is said to satisfy condition $D_{n}$ if $f\left(U_{n}(A)\right)$ is dense in $U_{n}(B)$ for every dense morphism $f: A \rightarrow B$.

Proposition 3.2. If $A$ satisfies condition $D_{n}$ then it satisfies $D_{n+1}$.

Proof. Let $f: A \rightarrow B$ be a dense morphism and take $b^{\prime}=\left(b, b_{n+1}\right)=$ $\left(b_{1}, \ldots, b_{n}, b_{n+1}\right) \in U_{n+1}(B)$. Let $I$ be the closed ideal generated by $b_{n+1}, C=B / I$ and $\pi: B \rightarrow C$ the natural projection. Then $\pi(b) \in U_{n}(C)$ and $\pi f: A \rightarrow C$ is dense. Given $\varepsilon>0$ there exists $a \in U_{n}(A)$ such that $\|\pi f(a)-\pi(b)\|<\varepsilon$. Then, there exist $y_{1}, \ldots, y_{n}$ in $B$ such that $\left\|f\left(a_{i}\right)-b_{i}-y_{i} b_{n+1}\right\|<\varepsilon(i=1, \ldots, n)$. By the density of $f(A)$ in $B$ there exist $x_{1}, \ldots, x_{n}, a_{n+1}$ in $A$ such that $\left\|f\left(x_{i}\right)-y_{i}\right\|<\varepsilon(i=1, \ldots, n)$ and $\left\|f\left(a_{n+1}\right)-b_{n+1}\right\|<\varepsilon$. Then $a^{\prime}=\left(a_{1}-x_{1} a_{n+1}, \ldots, a_{n}-x_{n} a_{n+1}, a_{n+1}\right) \in$ $U_{n+1}(A)$ and $\left\|f\left(a^{\prime}\right)-b^{\prime}\right\|<\varepsilon^{\prime}$. This proves that $A$ satisfies $D_{n+1}$.

A trivial consequence of the remarks above and the identification $\pi_{0}\left(U_{n}(A)\right)=$ $\left[X(A), C_{*}^{n}\right]$ is that $\operatorname{sr}(A)=\min \left\{n:\left[X(A), C_{*}^{n}\right] \rightarrow\left[F, C_{*}^{n}\right]\right.$ is onto for every hull $F$ of $X(A)\}$. The analogous property for dsr is the following.

Proposition 3.3. $\operatorname{dsr}(A)=\min \left\{n:\left[X(A), C_{*}^{n}\right] \rightarrow\left[F, C_{*}^{n}\right]\right.$ is onto for every $A$ convex $F \subset X(A)\}$.

Proof. Let $s$ be the number in the right-hand side. Suppose that $\operatorname{dsr}(A) \leqslant n$.

If $F$ is $A$-convex, we consider the dense morphism $A \rightarrow A_{F}=$ closure in $C(F)$ of $\hat{A} \mid F$. Then $f\left(U_{n}(A)\right)$ is dense in $U_{n}\left(A_{F}\right)$, which implies that $\left[X(A), C_{*}^{n}\right] \rightarrow\left[F, C_{*}^{n}\right]$ is onto. This proves that $s \leqslant \operatorname{dsr}(A)$. Conversely, if $f: A \rightarrow B$ is a dense morphism, $f\left(U_{n}(A)\right)$ is dense in $U_{n}(B)$ iff $\left[X(A), C_{*}^{n}\right] \rightarrow\left[X(B), C_{*}^{n}\right]$ is onto; but $X(B)$ is homeomorphic to $f^{*}(X(B))$ which is $A$-convex, by Proposition 2.1. Then $\operatorname{dsr}(A) \leqslant s$.

It is known that $\operatorname{sr}(A)=[\operatorname{dim} X(A) / 2]+1$ if $A$ is regular [4, 8]. The last proposition shows that $\operatorname{dsr}(A)=[\operatorname{dim} X(A) / 2]+1$ if every compact $F \subset X(A)$ is $A$-convex (see [10] for a generalization of this property). In particular, we get $\operatorname{sr}(A)=\operatorname{dsr}(A)$ if $A$ is regular.

THEOREM 3.4. Let $A$ be a n-generated Banach algebra, then $\operatorname{dsr}(A) \leqslant[n / 2]+1=$ $s_{n}$.

Proof. Let $a_{1}, \ldots, a_{n}$ be a system of generators of $A$ and suppose that $f: A \rightarrow B$ is a dense morphism; then $b_{1}=f\left(a_{1}\right), \ldots, b_{n}=f\left(a_{n}\right)$ generate $B$ and by the Main Theorem $U_{k}(A) \rightarrow U_{k}(B)$ has dense image for $k \geqslant s_{n}$.

Proposition 3.5. If $f: A \rightarrow B$ is a dense morphism then $\operatorname{dsr}(B) \leqslant \operatorname{dsr}(A)$.

Proof. Suppose that $\operatorname{dsr}(A) \leqslant n$. Then, if $g: B \rightarrow C$ is a dense morphism, $(g f)\left(U_{n}(A)\right)$ is dense in $U_{n}(C)$ and the inclusions $g\left(f\left(U_{n}(A)\right)\right) \subset g\left(U_{n}(B)\right) \subset U_{n}(C)$ show that $g\left(U_{n}(B)\right)$ is dense in $U_{n}(C)$.

We introduce two definitions for a homomorphism of Banach algebras $f: A \rightarrow B$. We say that $f(A)$ is an $n$-full subalgebra of $B$ if $f\left(A^{n}\right) \cap U_{n}(B)=f\left(U_{n}(A)\right)$. We say that $f$ is an $n$-full morphism if $f^{-1}\left(U_{n}(B)\right)=U_{n}(A)$. We say that $f$ is full when $f$ is 1 -full. Of course, if $f$ is $n$-full then $f(A)$ is $n$-full, but in general the converse fails. However, if $f$ is injective, both conditions are equivalent. 
Lemma 3.6 [9]. If $f: A \rightarrow B$ is a homomorphism of Banach algebras then $f: A \rightarrow B$ is $n$-full for every integer $n$ if and only if $f^{*}: X(B) \rightarrow X(A)$ is onto.

Lemma 3.7. If $f: A \rightarrow B$ is a dense full morphism then $f$ is $n$-full for every $n$.

The proof is routine.

Proposition 3.8. If $f: A \rightarrow B$ is a dense full morphism then $\operatorname{dsr}(A)=\operatorname{dsr}(B)$.

Proof. It suffices, by the last proposition, to see that $\operatorname{dsr}(A) \leqslant \operatorname{dsr}(B)$. But, using the characterization (Proposition 3.3) of $\operatorname{dsr}(A)$ and observing that $f^{*}: X(B) \rightarrow$ $X(A)$ is a homeomorphism which transforms, bijectively, $B$-convex subsets of $X(B)$ in $A$-convex subsets of $X(A)$, it is clear that $\operatorname{dsr}(A)=\mathrm{dsr}(B)$.

Corollary 3.9. $\mathrm{dsr}(A)=\mathrm{dsr}(P(X(A)))$.

Corollary 3.10. If $f: A \rightarrow B$ is a dense full morphism and $\operatorname{sr}(A)=\operatorname{dsr}(A)$ then $\operatorname{sr}(B)=\operatorname{dsr}(B)=\operatorname{sr}(A)$.

Proof. It is well known that $\operatorname{sr}(A) \leqslant \operatorname{sr}(B)$. Then $\operatorname{sr}(A) \leqslant \operatorname{sr}(B) \leqslant \operatorname{dsr}(B)=$ $\operatorname{dsr}(A)=\operatorname{sr}(A)$.

Remark 3.11. Swan [23] asked if a dense full subalgebra $A$ of $B$ has the same stable rank. The last corollary shows that this is, in fact, the case when $\operatorname{sr}(A)=$ $\operatorname{dsr}(A)$. But, as it was remarked before, we do not know if $\operatorname{sr}(A)=\operatorname{dsr}(A)$ in general.

THEOREM 3.12. Let $\left\{a_{1}, \ldots, a_{n}\right\}$ be a set of generators of $A$ such that its joint spectrum $\sigma(a)=\left\{\left(h\left(a_{1}\right), \ldots, h\left(a_{n}\right)\right): h \in X(A)\right\}$ has nonvoid interior (in $\left.C^{n}\right)$. Then $\operatorname{sr}(A) \geqslant s_{n}=[n / 2]+1$.

Proof. There is no loss of generality if we suppose that $B_{1}(0)=\left\{z \in C^{n}\right.$ : $\left.\sum_{i=1}^{n}\left|z_{i}\right|^{2} \leqslant 1\right\} \subset \sigma(a)$. Observe that $\sigma(a)$ is a polynomially convex compact subset of $C^{n}$ homeomorphic to $X(A)$ vía $\hat{a}=\left(\hat{a}_{1}, \ldots, \hat{a}_{n}\right): X(A) \rightarrow \sigma(a)$. Thus, we have a homomorphism $\varphi: A \rightarrow P(\sigma(a))$ sending $a_{i}$ into $z_{i}$ (the $i$ th-coordinate function). The theorem will be proved when we show that, for $n=2 k$ or $2 k+1, u=$ $\left(z_{1}, z_{3}, \ldots, z_{2 k-1}, p\right) \in U_{k+1}(P(\sigma(a)))$ is not reducible, where $p(z)=z_{1} z_{2}+z_{3} z_{4}$ $+\cdots+z_{2 k-1} z_{2 k}-1$. In fact, in this case $\left(a_{1}, a_{3}, \ldots, a_{2 k-1}, p(a)\right) \in U_{k+1}(A)$ is not reducible; otherwise, there should exist $b_{1}, \ldots, b_{k} \in A$ such that $\left(a_{2 i-1}+\right.$ $\left.b_{i} p(a)\right)_{i} \in U_{k}(A)$ and then $\left(z_{2 i-1}+\varphi\left(b_{i}\right) p\right)_{i} \in U_{k}(A)$ contradicting the irreducibility of $\left(z_{1}, z_{3}, \ldots, z_{2 k-1}, p\right)$. Suppose that $u$ is reducible. Then, by [8, Theorem 1.4], if $f(z)=\left(z_{1}, z_{3}, \ldots, z_{2 k-1}\right), f: \sigma(a) \rightarrow C^{k}$, and $Z_{p}=\{z \in \sigma(a): p(z)=0\}$, $f \mid Z_{p}: Z_{p} \rightarrow C_{*}^{k}$. Let $S=\left\{z \in B_{1}(0): z_{2 j}=\bar{z}_{2 j-1}, j=1, \ldots, k\right\}$. Then $f \mid Z_{p} \cap S$ can be extended to $S \rightarrow C_{*}^{k}$ and then the inclusion $i: S^{2 k-1} \rightarrow C_{*}^{k}$ admits an extension $B_{1}(0) \rightarrow C_{*}^{k}$, which is false, by elementary topology [21].

Corollary 3.13. $\operatorname{dsr}(P(X))=\operatorname{sr}(P(X))=s_{n}$ if $X$ is the n-polydisc $\Delta^{n}=\left\{z \in C^{n}\right.$ : $\left.\left|z_{i}\right| \leqslant 1(i=1, \ldots, n)\right\}$ or the $n$-ball $\left\{z \in C^{n}: \sum_{i=1}^{n}\left|z_{i}\right|^{2} \leqslant 1\right\}$ or $X$ is any compact subset of $C^{n}$ with nonvoid interior. 
Proof. It is a combination of Theorems 3.4 and 3.12 .

REMARK 3.14. $P(\Delta)$ was the first example of a Banach algebra $A$ such that $\operatorname{tsr}(A)-\operatorname{sr}(A)>0[7,15]$. Next, it was proved that for $A_{n}=P\left(\Delta^{n}\right), \operatorname{tsr}\left(A_{n}\right)=n+1$ and $\operatorname{sr}\left(A_{n}\right) \leqslant n$ [8, Theorems 2.1 and 3.1]. The last corollary shows that the difference $\operatorname{tsr}(A)-\operatorname{sr}(A)$ can be arbitrary large, because $\operatorname{tsr}\left(A_{n}\right)-\operatorname{sr}\left(A_{n}\right)=n-$ [n/2]. It is an open problem, however, if there exists $A$ such that $\operatorname{sr}(A)$ is finite and $\operatorname{tsr}(A)$ is infinite. We conjecture that $\operatorname{tsr}(A) \leqslant 2 \operatorname{sr}(A)$.

An interpolation result. Let $X$ be a compact subset of the closed disc $\Delta, A=P(\Delta)$ and $B=P(X)$. Then, the restriction $r: A \rightarrow B$ is a dense morphism and, if $\varepsilon>0$ and $f \in B$; i.e. $f(z) \neq 0$ for every $z \in \hat{X}$, there exists $F \in A$ such that $F(z) \neq 0$ for every $z \in \Delta$ and $\sup _{\hat{x}}|f-F|<\varepsilon$. If $X$ is hull, $F$ can be chosen such that $f=F \mid X$. It is a simple application of Theorem 3.4.

$A$ problem of Cartan. In a famous paper [3] H. Cartan studied the following problem: Let $\Omega$ be a certain domain of $C^{k}, H(\Omega)$ the algebra of all holomorphic functions on $\Omega$, and $f_{1}, \ldots, f_{m}$ an $m$-tuple of elements of $H(\Omega)$ without common zeros; does there exist an $m \times m$ invertible matrix $M$ with entries from $H(\Omega)$ such that the given $m$-tuple is the last column of $M$ ? This problem was later partially solved, in the context of Banach algebras (which means that $H(\Omega)$ is replaced by the algebra $A(\Omega)$ of continuous functions on $\bar{\Omega}$ which are holomorphic on $\Omega$ ) by Lin [16] and Sibony and Wermer [20]. Using the notations of this paper, Cartan's problem is to determine whether a given $a=\left(a_{1}, \ldots, a_{m}\right) \in U_{m}(A)$ belongs to the image of the map $t: \mathrm{GL}_{m}(A) \rightarrow U_{m}(A)$ defined by $\sigma \rightarrow$ last column of $\sigma$. The fibration properties of $t$ (see $\S 1$ and the references there) show that $a$ is in the image of $t$ if and only if some $b$ in the connected component of $a$ in $U_{m}(A)$ belongs to the image of $t$. Supose that $A$ is an $n$-generated Banach algebra. Then $t$ is onto, i.e. every element of $U_{m}(A)$ is the last column of an invertible matrix, for every $m \geqslant s_{n}$. In fact, by the Main Theorem $U_{m}(A)$ is connected and is onto. This improves the results of Lin and Sibony and Wermer in the case of finitely generated algebras.

On Oka's extension theorem. Let $\pi$ be a polynomial polyhedron (shortly, a $p$-polyhedron) in $C^{n} . \pi=\left\{z \in \Delta^{n}:\left|p_{j}(z)\right| \leqslant 1, j=1, \ldots, r\right\}$ for some polynomials $p_{1}, \ldots, p_{r}$ in $n$ variables.

Let $\phi: \pi \rightarrow C^{n+r}$ be the embedding $\phi(z)=\left(z, p_{1}(z), \ldots, p_{r}(z)\right)$ (where $z=$ $\left.\left(z_{1}, \ldots, z_{n}\right)\right)$. Then, $\phi$ is a homeomorphism of $\pi$ onto the subset of $\Delta^{n+r}$ defined by the equation

$$
z_{j+n}=p_{j}(z) \quad(j=1, \ldots, r) .
$$

Oka's extension theorem states that for every holomorphic function $f$ on a neighborhood of $\pi$ there exists a holomorphic function $F$ on a neighborhood of $\Delta^{n+r}$ such that

$$
F\left(z, p_{1}(z), \ldots, p_{r}(z)\right)=f(z) \quad(z \in \pi) .
$$

( $F$ is called an Oka extension of $f$.)

THEOREM 3.15. Let $f$ be a $k$-tuple of holomorphic functions on a neighborhood of $\pi$ such that $f(\pi) \subset C_{*}^{k}$. Suppose that $k \geqslant s_{n}$. Then, there exists an Oka extension $F$ : $U \rightarrow C_{*}^{k}$ for some neighborhood $U$ of $\Delta^{n+r}$. 
PROOF. Let $\varepsilon>0$ and $\pi_{\varepsilon}=\left\{z \in \Delta_{1+\varepsilon}^{n}:\left|p_{j}(z)\right| \leqslant 1+\varepsilon, 1 \leqslant j \leqslant r\right\}$ (where $\Delta_{1+\varepsilon}^{n}$ $\left.=\left\{z \in C^{n}:\left|z_{j}\right| \leqslant 1+\varepsilon, 1 \leqslant j \leqslant r\right\}\right)$ such that $\pi_{\varepsilon}$ is contained in the domain of $f$ and $f\left(\pi_{\varepsilon}\right) \subset C_{*}^{k}$. By Oka's theorem there exist holomorphic functions on a neighborhood of $\Delta_{1+\varepsilon}^{n+r}, G_{1}, \ldots, G_{k}$ such that

$$
G\left(z, p_{1}(z), \ldots, p_{r}(z)\right)=f(z) \quad\left(z \in \pi_{\varepsilon}\right) .
$$

where $G=\left(G_{1}, \ldots, G_{k}\right)$. Let $A=P\left(\Delta_{1+\varepsilon}^{n+r}\right), I$ the closed ideal of $A$ generated by $\left\{z_{n+1}-p_{1}, \ldots, z_{n+r}-p_{r}\right\}$, and $\varphi: A \rightarrow A / I$ the natural projection. Then $\varphi(G) \in$ $U_{k}(A / I)$ because $X(A(I))$ is the hull of $I$ and $G \mid$ hull $(I)=f \mid$ hull $(I)$ never assumes the value $0=(0, \ldots, 0) \in C^{k}$. Observe that $A / I$ is generated by $\varphi\left(z_{1}\right), \ldots, \varphi\left(z_{n}\right)$. Then, for $k \geqslant s_{n} \varphi\left(U_{k}(A)\right)=U_{k}(A / I)$, by the Main Theorem, part (1). In particular, there exists $F \in U_{k}(A)$ such that $\varphi(F)=\varphi(G)$; in other words $F=\left(F_{1}, \ldots, F_{k}\right)=\left(G_{1}+H_{1}, \ldots, G_{k}+H_{k}\right)$ for some $H_{1}, \ldots, H_{k}$ in $I$. Thus, $F$ is holomorphic on a neighborhood of $\Delta^{n+r}, \quad F\left(\Delta^{n+r}\right) \subset C_{*}^{k}$ and $F\left(z, p_{1}(z), \ldots, p_{r}(z)\right)=G\left(z, p_{1}(z), \ldots, p_{r}(z)\right)=f(z)$ for $z \in \pi$.

On a problem of Swan. In [23] Swan asked if the existence of a dense full morphism $f: A \rightarrow B$ implies that $\operatorname{sr}(A)=\operatorname{sr}(B)$. It is easily proved that $\operatorname{sr}(A) \leqslant$ $\operatorname{sr}(B)$ and we have seen that, for $A$ such that $\operatorname{sr}(A)=\operatorname{dsr}(A)$, the answer is affirmative. We conjecture that Swan's question always has an affirmative answer but we have been unable to prove this. However we can show that $\operatorname{sr}(B)$ cannot exceed $\operatorname{sr}(A)$ by more than 2 . We begin with

THEOREM 3.16. $\operatorname{sr} P(X(A)) \leqslant \operatorname{sr}(A)+2$.

Proof. Let $n=\operatorname{sr}(A)$. By [8, theorem 1.4] it suffices to prove that for every $b \in P(X(A))$ and every map $f: Z_{b} \rightarrow C_{*}^{n+2}$ there exists a continuous extension $F$ of $f, \quad F: X(A) \rightarrow C_{*}^{n+2}$. Given a map $f: Z_{b} \rightarrow C_{*}^{n+2}$, consider an extension $f:$ $X(A) \rightarrow C^{n+2}$ and a compact neighborhood $V$ of $Z_{b}$ such that $f(V) \subset C_{*}^{n+2}$. Let $\delta=\min \{|b(h)|: h \in X(A) \backslash V\}>0, a \in A$ be such that $\|\hat{a}-b\|<\delta / 4$ and $R=$ $\{h \in X(A):|h(a)| \leqslant \delta / 2\}$. We have $Z_{b} \subset R \subset V$. Consider the algebra $B=$ $C(\Delta, A)$ and the element $\theta: \omega \rightarrow a-(\delta / 2) \omega(\omega \in \Delta)$ of $B$. It is known that $\operatorname{sr}(B) \leqslant \operatorname{sr}(A)+2[5]$, so we get a surjective mapping $\left[\Delta \times X(A), C_{*}^{n+2}\right] \rightarrow$ $\left[Z_{\theta}, C_{*}^{n+2}\right]$ (where $Z_{\theta}=\{(h, \omega) \in X(A) \times \Delta: h(\theta(\omega))=0\}$ ). We prove now that the first projection $p:(h, \omega) \rightarrow h$ defines a homeomorphism from $Z_{\theta}$ onto $R$; the continuity of $p$ is obvious; if $h \in R$ then $h(a)=\lambda$ with $|\lambda| \leqslant \delta / 2$ and $\omega=(2 / \delta) \lambda$ $\in \Delta ;(h, \omega) \in Z_{\theta}$ and, of course $p(h, \omega)=h$, which proves that $p$ is surjective, if $\left(h_{0}, \omega_{1}\right)$ and $\left(h_{0}, \omega_{2}\right)$ belongs to $Z_{\theta}$ then $h_{0}(a)=(\delta / 2) \omega_{1}=(\delta / 2) \omega_{2}$ so $\omega_{1}=\omega_{2}$. Finally, $R$ being homeomorphic to $Z_{\theta}$ we get the surjection

$$
\left[\Delta \times X(A), C_{*}^{n+2}\right] \rightarrow\left[R, C_{*}^{n+2}\right]
$$

which becomes, using the contractibility of $\Delta$,

$$
\left[X(A), C_{*}^{n+2}\right] \rightarrow\left[R, C_{*}^{n+2}\right] .
$$

This shows that $f \mid R: R \rightarrow C_{*}^{n+2}$ admits an extension $F: X(A) \rightarrow C_{*}^{n+2}$ (see [8, Theorem 1.4]), which proves the theorem. 
RemarK 3.17. It is not known if $\operatorname{sr}(C(\Delta, A)) \leqslant 1+\operatorname{sr}(A)$. If this inequality would hold then the proof above shows that $\operatorname{sr}(P(X(A))) \leqslant 1+\operatorname{sr}(A)$.

Corollary 3.18. If $f: A \rightarrow B$ is a dense full morphism then $\operatorname{sr}(A) \leqslant \operatorname{sr}(B) \leqslant$ $\operatorname{sr}(A)+2$.

Proof. It is well known that $P(X(A))$ is isomorphic to $P(X(B))$ and then $\operatorname{sr}(B) \leqslant \operatorname{sr}(P(X(B)))=\operatorname{sr}(P(X(A))) \leqslant \operatorname{sr}(A)+2$.

\section{REFERENCES}

1. A. Andreotti and R. Narasimhan, A topological property of Runge pairs, Ann. of Math. (2) 76 (1962), 499-509.

2. H. Bass, K-theory and stable algebra, Publ. Math. Inst. Hautes Etudes Sci. no. 22, 1964, pp. 5-60.

3. H. Cartan, Sur les matrices holomorphes de $n$ variables complexes, J. Math. Pures Appl. 19 (1940), $1-26$.

4. G. Corach and A. R. Larotonda, Stable range in Banach algebras, J. Pure Appl. Algebra 32 (1984), 289-300.

5. __ A stabilization theorem for Banach algebras, J. Algebra 101 (1986), 433-449.

6. Unimodular matrices in Banach algebra theory, Proc. Amer. Math. Soc. 96 (1986), 473-477.

7. G. Corach and F. D. Suárez, Stable rank in holomorphic function algebras, Illinois J. Math. 29 (1985).

8. Extension problems and stable rank in commutative Banach algebras, Topology Appl. 21 (1985), 1-18.

9. ___ Extension of characters in commutative Banach algebras, Studia Math. 85 (1987), 199-202.

10. Generalized rational convexity in Banach algebras (preprint).

11. T. Duchamp and E. L. Stout, Maximum modulus sets, Ann. Inst. Fourier (Grenoble) 31 (1981), $37-69$.

12. T. W. Gamelin, Uniform algebras, Chelsea, New York, 1984.

13. R. Herman and L. N. Vaserstein, The stable range of $C^{*}$ algebras, Invent. Math. 77 (1984), 553-555.

14. S. T. Hu, Homotopy theory, Academic Press, New York, 1959.

15. P. Jones, D. Marshall and T. Wolff, The stable rank of the disc algebra, Proc. Amer. Math. Soc. 96 (1986), 603-604.

16. V. Ya Lin, Holomorphic fiberings and multivalued functions of elements of a Banach algebra, Funct. Anal. Appl. 7 (1973), 122-128.

17. E. Michael, Convex structures and continuous selections, Canad. J. Math. 4 (1959), 556-575.

18. C. E. Rickart, General theory of Banach algebras, Van Nostrand, Princeton, N. J., 1960.

19. M. Rieffel, Dimension and stable rank in the K-theory of $C^{*}$ algebras, Proc. London Math. Soc. (3) 46 (1983), 301-333.

20. N. Sibony and J. Wermer, Generators for $A(\Omega)$, Trans. Amer. Math. Soc. 194 (1974), 103-114.

21. E. H. Spanier, Algebraic topology, McGraw-Hill, New York, 1966.

22. E. L. Stout, The theory of uniform algebras, Bodgen \& Quigley, Tanytown-on-Hudson, N. Y., 1971.

23. R. S. Swan, Topological examples of projective modules, Trans. Amer. Math. Soc. 230 (1977), $201-234$.

24. J. L. Taylor, Topological invariants of the maximal ideal space of a Banach algebra, Adv. in Math. 19 (1976), 149-206.

25. L. N. Vaserstein, Stable range of rings and dimensionality of topological spaces, Funct. Anal. Appl. 5 (1971), 102-110.

Instituto Argentino de Matemática, Viamonte 1636, $2^{\circ}$ " A”, 1055 Buenos Aires, Argentina 